\title{
Refractive index sensor based on asymmetrical Mach-Zehnder interferometer with step-like tapers (Withdrawal Notice)
}

Feng Xia, Yong Zhao, Hai-feng Hu, Mao-qing Chen, Yanan Zhang

Feng Xia, Yong Zhao, Hai-feng Hu, Mao-qing Chen, Ya-nan Zhang, "Refractive index sensor based on asymmetrical Mach-Zehnder interferometer with step-like tapers (Withdrawal Notice)," Proc. SPIE 10323, 25th International Conference on Optical Fiber Sensors, 1032328 (23 April 2017); doi: 10.1117/12.2263255

Event: 25th International Conference on Optical Fiber Sensors, 2017, Jeju, Korea, Republic of 


\section{Refractive index sensor based on asymmetrical Mach-Zehnder interferometer with step-like tapers (Withdrawal Notice)}

Proc. SPIE 10323, 1032328 (2017)

Online Publication Date: 23 April 2017

Withdrawn from Publication: 17 May 2017

Conference Date: 24-28 April 2017

Conference Location: Jeju, Korea, Republic of

Conference Title: 25th International Conference on Optical Fiber Sensors

Conference Chairs: Youngjoo Chung, Wei Jin, Byoungho Lee, John Canning, Kentaro Nakamura, Libo Yuan

Feng Xia, Yong Zhao, Hai-feng Hu, Mao-qing Chen, Ya-nan Zhang

Northeastern Univ. (China)

This paper has been withdrawn by the publisher at the request of the authors. 